\title{
An archaeal order with multiple minichromosome maintenance genes
}

\begin{abstract}
Correspondence
James P. J. Chong

jpjc1@york.ac.uk
\end{abstract}

Received 24 November 2009

Revised 12 January 2010

Accepted 29 January 2010

\author{
Alison D. Walters and James P. J. Chong \\ Department of Biology (Area 5), PO Box 373, University of York, York YO10 5YW, UK
}

In eukaryotes, a complex of six highly related minichromosome maintenance (MCM) proteins is believed to function as the replicative helicase. Until recently, systems for exploring the molecular mechanisms underlying eukaryotic MCM function have been biochemically intractable. To overcome this, molecular studies of MCM function have been carried out using MCM homologues from the archaea. Archaeal MCM systems studied to date possess a single functional MCM, which forms a homohexameric complex that displays DNA binding, ATPase and helicase activities. We have identified an archaeal order that possesses multiple MCM homologues. BLAST searches of available Methanococcales genomes reveal that members of this order possess between two and eight MCM homologues. Phylogenetic analysis suggests that an ancient duplication in the Methanococcales gave rise to two major groups of MCMs. One group contains Methanococcus maripaludis S2 McmD and possesses a conserved C-terminal insert similar to one observed in eukaryotic MCM3, while the other group contains McmA, -B and -C. Analysis of the genome context of MCMs in the latter group indicates that these genes could have arisen from phagemediated events. When co-expressed in Escherichia coli, the four MCMs from M. maripaludis copurify, indicating the formation of heteromeric complexes in vitro. The presence of homologues from both groups in all Methanococcales indicates that there could be functionally important differences between these proteins and that Methanococcales MCMs may therefore provide an interesting additional model for eukaryotic MCM function.

\section{INTRODUCTION}

The archaea make up a third domain of life that is best known for including a large number of 'extremophilic' organisms. This group of prokaryotes was first identified as being distinct from bacteria by differences in their $16 \mathrm{~S}$ rRNA sequences (Woese \& Fox, 1977). Methanogens were the first organisms to be identified as archaea and classified as a separate domain (Woese \& Fox, 1977). Five orders of methanogens exist: Methanobacteriales, Methanococcales, Methanomicrobiales, Methanosarcinales and Methanopyrales (Thauer, 1998). Complete genome sequences for representatives of all of these orders are available. Initial analyses of the Methanocaldococcus (previously Methanococcus) jannaschii genome have revealed that the archaea have many metabolic characteristics in common with bacteria, but that the genes used for information processing

Abbreviations: GST, glutathione S-transferase; MCM, minichromosome maintenance; $\mathrm{ML}$, maximum-likelihood; $\mathrm{NJ}$, neighbour-joining.

A supplementary table, showing the results of a codon-based comparison of synonymous changes in MCM sequences from the same species, and two supplementary figures, showing an alignment of eukaryotic MCMs, and the genome context of McmA and McmD genes in the Methanococcales, are available with the online version of this paper. are more similar to equivalent systems in eukaryotes (Bult et al., 1996; Edgell \& Doolittle, 1997). The observation that archaeal information-processing systems are similar to but simpler than those in eukaryotes, along with the thermostable nature of proteins from many of these organisms, has led to a number of archaeal species being used as models for a variety of eukaryotic processes (Tye, 2000).

DNA replication is a good example of how a eukaryotic process is simplified in archaea. The heterohexameric minichromosome maintenance (MCM) complex in eukaryotes is believed to be the genomic DNA helicase; that is, the protein that is responsible for unwinding genomic DNA prior to replication (Labib et al., 2000, 2001; Prokhorova \& Blow, 2000). A range of different MCM complexes have been isolated from eukaryotic systems. The heteromeric nature of these complexes and the requirement for all six proteins has posed a significant hurdle to a comprehensive biochemical characterization of the MCM helicase. In archaea that have been characterized to date, a single MCM protein forms a homohexameric complex that possesses ATP-dependent DNA helicase activity. MCM complexes from a number of species (most notably Sulfolobus and Methanothermobacter) have been characterized and have provided insight into the mechanisms that govern helicase activity in these proteins. Motifs essential for 
MCM function have been identified using ATP hydrolysis, DNA binding and DNA helicase activity assays (Barry et al., 2007; Carpentieri et al., 2002; Chong et al., 2000; Jenkinson \& Chong, 2006; Kasiviswanathan et al., 2004; Kelman et al., 1999; Poplawski et al., 2001; Shechter et al., 2000).

The genome sequence of Methanocaldococcus jannaschii has revealed that in contrast to the single MCM found in other archaea, this organism has multiple, apparently functional MCM homologues (Bult et al., 1996). Analysis of the genome sequence of Methanococcus maripaludis S2 has revealed that it also contains multiple MCMs (Hendrickson et al., 2004; Walters \& Chong, 2009). The four M. maripaludis S2 MCM homologues are distributed across the genome and are assigned ORF numbers that we have further supplemented with gene names (indicated in parentheses) as MMP0030 $(m c m A)$, MMP0470 $(m c m B)$, MMP0748 $(m c m C)$ and MMP1024 $(\mathrm{mcmD})$. Sequence analysis of additional Methanococcales genomes has shown that all of them possess multiple MCM homologues. Our results suggest that the MCM genes underwent an ancient duplication in the order Methanococcales, probably before the current species evolved. In addition, we present evidence that two distinct classes of MCM exist in the Methanococcales, one of which has been duplicated through the action of a mobile element that can still be observed on a cryptic plasmid found in these species; the other has similarities to eukaryotic MCM3. Coexpression of recombinant MCMs from M. maripaludis S2 allowed co-purification of all four proteins, indicating that they form one or more heteromeric complexes. Our data support the hypothesis that the Methanococcales are unique among the archaea in possessing multiple functional MCMs and may therefore be a good model for understanding eukaryotic MCM interactions.

\section{METHODS}

MCM identification. To identify MCM homologues, the MMP0030 $(\mathrm{McmA})$ protein was used as the query sequence in a BLASTP (Altschul et al., 1997) search against the NCBI non-redundant database (Pruitt et al., 2007) with a cut off value of $1 \mathrm{e}^{-20}$.

\begin{abstract}
Phylogenetic analyses. A multiple sequence alignment containing 31 potentially functional Methanococcales MCM homologues (listed in Table 1) plus an outgroup of Methanobacteria (Methanothermobacter thermautotrophicus, Methanobacterium stadtmanae and Methanobrevibacter smithii) and Methanopyrus kandleri was constructed in CLUSTAL_X (Thompson et al., 1997). The alignment of 35 sequences was edited using Gblocks (Castresana, 2000). This edited alignment was used to generate a phylogenetic tree using both neighbour-joining (NJ) and maximum-likelihood (ML) methods. ML phylogenetic analyses were performed using PHYML (Guindon \& Gascuel, 2003) with the JTT substitution model applied (Jones et al., 1992). One hundred bootstrap replicates were generated for ML analysis and 1000 replicates for the NJ analysis. The two different methods gave the same basic tree topology.
\end{abstract}

Identification of homologous flanking genes. Related gene contexts of MCMs were initially identified using genome comparison tools at http://theseed.uchicago.edu/FIG/index.cgi (Overbeek et al., 2005).

Assembly of contextual maps. The contextual arrangement of genes surrounding Methanococcales MCM homologues was visualized using Lasergene (Burland, 2000). Homologous genes were identified in other genomes from the order using BLASTP (Altschul et al., 1997), using a cut-off of $1 \mathrm{e}^{-20}$.

Identification of repeat sequences. DNA sequences in the region of MCM genes were examined for repeat sequences using MEME (Bailey \& Elkan, 1994) with the following parameters: any number of repetitions, minimum width 6 , maximum width 50 , up to 5 motifs found.

Expression and purification of $\boldsymbol{M}$. maripaludis S2 MCMs. The four M. maripaludis S2 MCMs were amplified by PCR from genomic DNA. $m c m A$ and $m c m C$ were cloned into pCDF-2 Ek/LIC (Novagen) with a T7-tag adaptor (Novagen) using ligation-independent cloning such that $m c m A$ had an N-terminal hexa-histidine tag and $m c m C$ had an N-terminal T7 tag. $m c m B$ and $m c m D$ were cloned into pET32 EkLIC (Novagen) with a glutathione $S$-transferase (GST) adaptor such that $m c m B$ had an N-terminal Trx.hexa-histidine.S tag and $m c m D$ had an N-terminal GST tag. The expression constructs were co-transformed into Rosetta BL21(DE3) Escherichia coli cells (Novagen). Cells were grown with shaking (200 r.p.m.) at $37^{\circ} \mathrm{C}$ until the $\mathrm{OD}_{600}$ reached 0.8

Table 1. Methanococcales MCM homologues identified by BLASTP searches using MMP0030 (McmA) as the query sequence

The genome sizes of all organisms are similar, but the number of MCMs ranges from two to eight. Methanocaldococcus jannaschii and M. vannielii both possess truncated MCM homologues that lack motifs essential for function (ORF numbers for truncated MCMs are shown in parentheses).

\begin{tabular}{|c|c|c|c|c|}
\hline Organism & $\begin{array}{l}\text { Genome } \\
\text { size }(\mathrm{Mb})\end{array}$ & $\begin{array}{l}\text { Total MCM } \\
\text { homologues }\end{array}$ & $\begin{array}{l}\text { Number of potentially } \\
\text { functional MCMs }\end{array}$ & MCM ORF numbers \\
\hline M. maripaludis $\mathrm{S} 2$ & 1.66 & 4 & 4 & МMP0030; ММР0470; ММР0748; МMP1024 \\
\hline M. maripaludis C5 & 1.81 & 4 & 4 & MmarC5_0570; MmarC5_0643; MmarC5_1119; MmarC5_1650 \\
\hline M. maripaludis C6 & 1.7 & 8 & 8 & $\begin{array}{l}\text { MmarC6_0030; MmarC6_0108; MmarC6_0133; MmarC6_0420; } \\
\text { MmarC6_0438; MmarC6_0919; MmarC6_1635; MmarC6_1837 }\end{array}$ \\
\hline M. maripaludis $\mathrm{C} 7$ & 1.8 & 4 & 4 & MmarC7_0027; MmarC7_0065; MmarC7_0266; MmarC7_1027 \\
\hline M. aeolicus Nankai-3 & 1.6 & 3 & 3 & Maeo_0490; Maeo_1144; Maeo_1226 \\
\hline M. vannielii SB & 1.7 & 4 & 3 & Mevan_0267; Mevan_0348; Mevan_1027 (Mevan_1640) \\
\hline M. voltae A3 & 1.86 & 2 & 2 & MvolDRAFT_0034; MvolDRAFT_0575 \\
\hline $\begin{array}{l}\text { Methanocaldococcus } \\
\text { jannaschii }\end{array}$ & 1.76 & 4 & 3 & Mjan_0363; Mjan_0961; Mjan_1489 (Mjan_ECL13) \\
\hline
\end{tabular}


and then chilled on ice for 20 min before induction with $0.1 \mathrm{mM}$ IPTG. Cultures were incubated for a further $24 \mathrm{~h}$ at $12{ }^{\circ} \mathrm{C}$ with 200 r.p.m. shaking before cells were harvested. Cells were resuspended in $2 \mathrm{ml} \mathrm{g}^{-1}$ lysis buffer ( $25 \mathrm{mM}$ Tris, $\mathrm{pH} 8.0,150 \mathrm{mM} \mathrm{NaCl}, 10 \%$, v/v, glycerol, $1 \mathrm{mM}$ PMSF, $1 \mu \mathrm{g}$ leupeptin $\mathrm{ml}^{-1}, 1 \mu \mathrm{g}$ pepstatin $\mathrm{ml}^{-1}, 1 \mu \mathrm{g}$ aprotinin $\mathrm{ml}^{-1}$ ), supplemented with $0.75 \mathrm{mg}$ lysozyme $\mathrm{ml}^{-1}$ and $5 \mu \mathrm{g}$ DNase $\mathrm{ml}^{-1}$. Cells were lysed by sonication and the lysate was clarified by centrifugation at $50000 \mathrm{~g}$ for $30 \mathrm{~min}$.

$\mathrm{McmD}$ and associated proteins were purified from 1.51 culture using $250 \mu$ lglutathione-Sepharose beads (GE Healthcare). After the addition of cell lysate, beads were washed with 10 column volumes of lysis buffer (as above) and eluted in $50 \mathrm{mM}$ Tris, $\mathrm{pH} 8.0,150 \mathrm{mM} \mathrm{NaCl}, 40 \mathrm{mM}$ reduced glutathione. For the multi-step purification, MCM protein was purified from $4.5 \mathrm{l}$ culture using nickel-Sepharose beads (GE Healthcare), eluted in $25 \mathrm{mM}$ Tris, $\mathrm{pH} 8.0,150 \mathrm{mM} \mathrm{NaCl}, 10 \%$, v/v, glycerol, $500 \mathrm{mM}$ imidazole. Elution fractions totalling $2 \mathrm{ml}$ from the nickel-Sepharose purification were pooled and further purified by sizeexclusion chromatography using a Superdex 200 16/60 (GE Healthcare) column in $25 \mathrm{mM}$ Tris, $\mathrm{pH} 8.0,150 \mathrm{mM} \mathrm{NaCl}, 10 \%, \mathrm{v} / \mathrm{v}$, glycerol at a flow rate of $1 \mathrm{ml} \mathrm{min}^{-1}$. Fractions containing MCMs were identified by SDS-PAGE, pooled and immediately loaded onto a $1 \mathrm{ml}$ Source Q column (GE Healthcare), then eluted in $25 \mathrm{mM}$ Tris, $\mathrm{pH}$ 8.0, $10 \%$, v/v, glycerol, $1 \mathrm{mM}$ PMSF, $1 \mu \mathrm{g}$ leupeptin $\mathrm{ml}^{-1}, 1 \mu \mathrm{g}$ pepstatin $\mathrm{ml}^{-1}, 1 \mu \mathrm{g}$ aprotinin $\mathrm{ml}^{-1}$ over a $30 \mathrm{ml} 50-1000 \mathrm{mM} \mathrm{NaCl}$ gradient at a flow rate of $1 \mathrm{ml} \mathrm{min}{ }^{-1}$. Elution fractions were separated by SDS-PAGE and analysed by Western blotting using anti-His $(\mathrm{McmA})$, anti-S $(\mathrm{McmB})$, anti-T7 $(\mathrm{McmC})$ and anti-GST $(\mathrm{McmD})$ antibodies.

\section{RESULTS}

\section{Identification of MCM homologues in Methanococcales}

Complete genome sequences exist for $M$. maripaludis strains S2, C5, C6 and C7, Methanocaldococcus jannaschii, Methanococcus aeolicus Nankai-3 and Methanococcus vannielii SB (http://www.ncbi.nlm.nih.gov/sites/genome/). Most of the Methanococcus voltae $\mathrm{A} 3$ genome is also complete, although a single contiguous sequence is not available. BLAST searches were carried out against each of the eight Methanococcales genomes using $\mathrm{McmA}$ from $M$. maripaludis S2 as the query sequence. Hits with an expect score of $1 \mathrm{e}^{-20}$ or better were considered to be reliable MCM homologues. Results showed that all of the Methanococcales genomes contain multiple MCMs. MCM homologue numbers range from two (in $M$. voltae A3) to eight (in M. maripaludis C6) (Table 1). Genome sizes are similar in all eight organisms, indicating that there have been no recent large genome duplications that have led to, for example, the doubling of MCM number in M. maripaludis C6 compared with M. maripaludis S2.

\section{Multiple sequence alignments of Methanococcales MCMs}

Multiple sequence alignments were generated from all the MCM homologues identified in Methanococcus genomes along with MCM homologues from three Methanobacteria. The alignment shows that of the 33 Methanococcales MCM sequences, 30 contain the known motifs required for MCM function (Fig. 1). Of those not possessing all the required motifs, two are truncated and do not contain the arginine finger required for ATP hydrolysis. One of these truncated proteins is found on an extra-chromosomal element in Methanocaldococcus jannaschii (MJ_ECL13) and the other is on the main chromosome of $M$. vannielii (Mevan_1640). The third protein showing significant changes in the normally conserved MCM motifs was ORF 0961 of Methanocaldococcus jannaschii. This protein contains a large insertion between the first two cysteines of the zinc finger, and lacks the second pair of cysteines.

Interestingly, a highly conserved 20 amino acid insertion is found in one MCM homologue from each of the eight Methanococcales analysed. This insertion is not present in any other archaeal MCMs and is particularly interesting because analysis of the six eukaryotic MCM sequences has shown that an insertion of similar size and in an equivalent position is unique to MCM3 (Supplementary Fig. S1). Evidence suggests that MCM3 may play a regulatory role in the eukaryotic MCM complex (Lin et al., 2008; Shi et al., 2007; Takei et al., 2002), so it is possible that this insertion plays a role in regulating $\mathrm{MCM}$ activity in the Methanococcales. The C-terminal domain of other archaeal MCMs has been shown to regulate MCM activity in vitro (Grainge et al., 2003; Jenkinson \& Chong, 2006), and therefore any insertions in the $C$ terminus could potentially alter its regulatory activity. The fact that there is an MCM homologue with a similar insertion in each of the eight Methanococcales genomes suggests that this protein plays a role different from the other Methanococcales MCMs, and indeed MCMs found in other archaea.

\section{Phylogenetic analysis of Methanococcales MCMs}

In order to gain some insight into the evolution of MCMs in the Methanococcales, phylogenetic analysis was carried out on all of the full-length Methanoccocales MCM sequences available (Fig. 2). The tree shows that the Methanococcales MCMs are more closely related to each other than they are to members of the outgroup, which is representative of other methanogen MCMs. The tree also shows that there have been multiple duplications of MCMs within the Methanococcales. An ancient duplication that pre-dates the divergence of the Methanococcales into separate species appears to have given rise to two major groups of MCMs. One of these groups contains one MCM homologue from each of the eight Methanococcales species analysed. This group contains $\mathrm{McmD}$ from M. maripaludis S2 and has therefore been named the 'McmD' group (Fig. 2). All eight members of this group contain the conserved 20 amino acid C-terminal insert identified above. The $\mathrm{McmD}$ group is strongly supported by high bootstrap values for both NJ and ML analyses. The second group produced from the ancient duplication has undergone further duplications to generate several subgroups of MCMs. One of these subgroups includes a single homologue from each of the eight Methanococcales species analysed. This subset of eight proteins includes McmA from M. maripaludis $\mathrm{S} 2$ and has therefore been named the ' $\mathrm{McmA}$ ' group. There are three subgroups related to the McmA group 
Zinc-finger
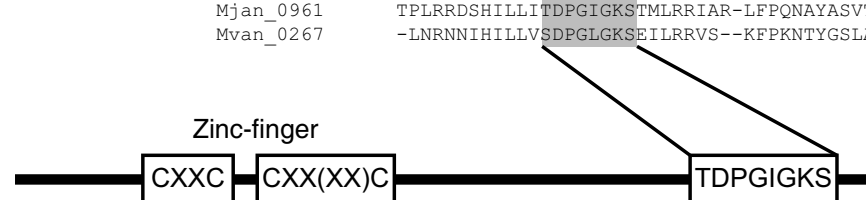
CXXC

Maeo_1226 Mjan 0363 MMP $\$ 2003$ Mvan 1027 Maeo_-1144 Mjan-1489 MMP S2 0748 MMP S2 0470 MMP S2 1024 Maeo- 0490 Mvol-0575

\begin{tabular}{l}
\hline \\
\\
30 \\
74 \\
24
\end{tabular}

Fig. 1. Summary of observations from a multiple sequence alignment of Methanococcales MCMs. Conserved motifs important for function are represented by boxes containing consensus sequences. $\mathrm{H} 2 \mathrm{i}$, helix-2 insert; $\mathrm{PS} 1 \mathrm{BH}$, pre-sensor $1 \beta$-hairpin; $\mathrm{R}$-finger, arginine finger. Alignments for each conserved motif are shown in full. A 20 amino acid insert is conserved at the $\mathrm{C}$ terminus of one MCM homologue in all organisms analysed, represented by McmD in M. maripaludis S2. MCMs from M. maripaludis C5, C6 and C7 are not included in the expanded alignments, but contain the same conserved motifs as M. maripaludis S2. 


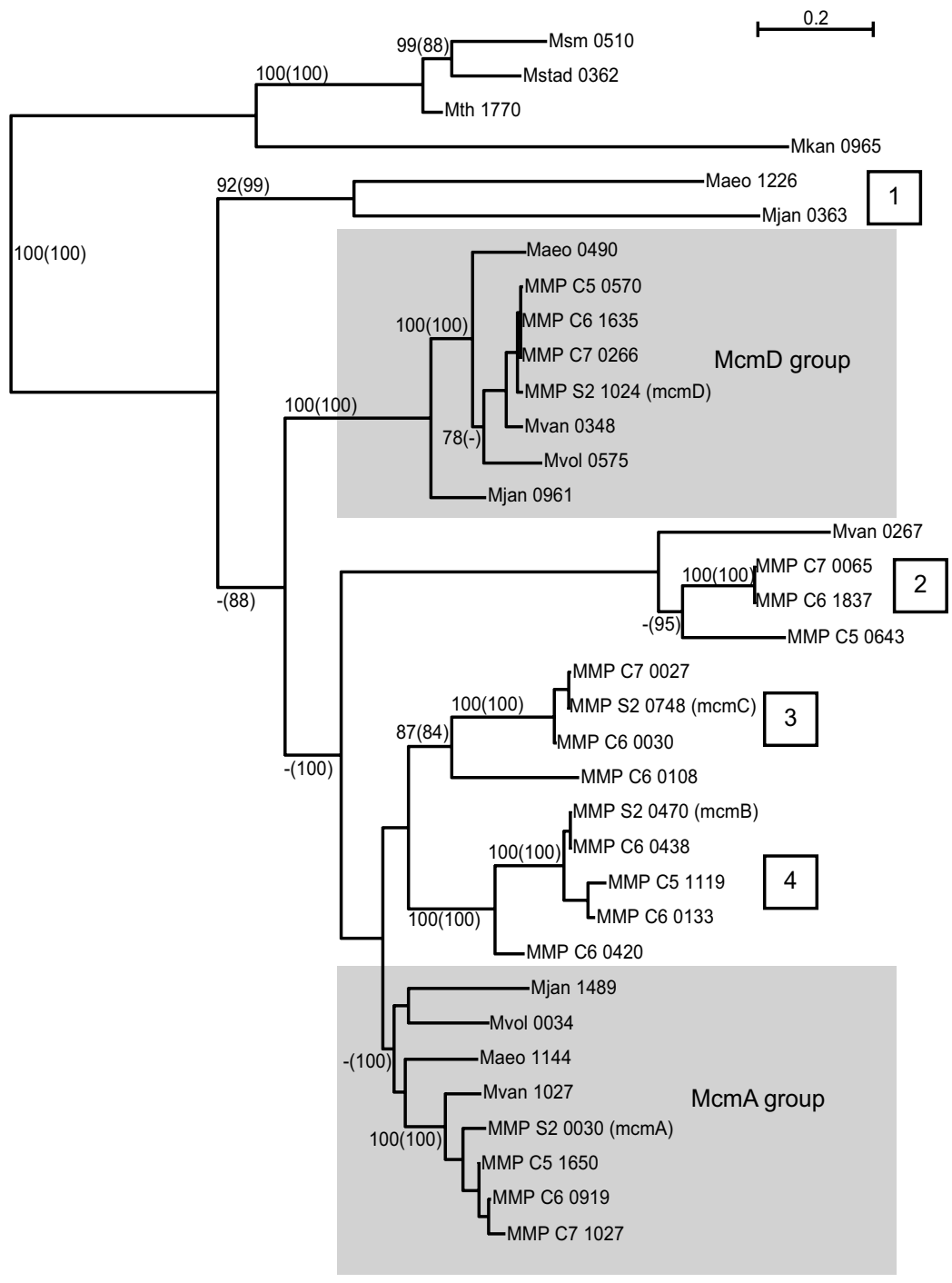

Fig. 2. ML phylogeny of the Methanococcales MCMs. ML and NJ trees were drawn using the same 272 amino acid alignment. A total of 1000 bootstraps were performed for the $\mathrm{NJ}$ tree and 100 bootstraps for the ML tree. Percentage bootstrap values are shown for the major groups recovered using $\mathrm{ML}$ and $\mathrm{NJ}$ (indicated in parentheses) analyses. Only bootstrap values over $75 \%$ are shown. The McmA and McmD groups are highlighted by shaded boxes. Other Methanococcales groups are labelled 1-4. MMP, M. maripaludis; Msm, Methanobrevibacter smithii; Mstad, Methanobacterium stadtmanae; Mth, Methanothermobacter thermautotrophicus; Mkan, Methanopyrus kandleri; Mjan, Methanocaldococcus jannaschii; Maeo, Methanococcus aeolicus; Mvol, M. voltae; Mvan, M. vannielii.

(Fig. 2, groups 2, 3 and 4) that have been produced by a number of further duplications, but these duplications are not common to every species.

As an additional measure of whether the multiple MCM genes in each Methanococcales species were likely to retain function, we measured selective pressure on the genes through the pairwise comparison of $d \mathrm{~S} / d \mathrm{~N}$ ratios of genes in each species. Our codon-based analysis indicated that within all individual species synonymous mutations were favoured over non-synonymous changes. Together with the conservation of all known motifs required for MCM activity, this result is consistent with the notion that all MCM homologues could be functional (Supplementary Table S1).

\section{Genome context analysis of Methanococcales MCMs}

The phylogenetic tree (Fig. 2) shows that there have been several duplications of MCMs within the Methanococcales. To investigate the nature of these duplications, the genome context of all full-length Methanococcales MCMs was compared (Fig. 3 and Supplementary Fig. S2). The comparison showed that all of the MCMs outside the $\mathrm{McmA}$ and $\mathrm{McmD}$ groups (Fig. 2) are found close to an integrase homologue (Fig. 3, yellow). There is an 83 amino acid ORF that is present upstream of the integrase gene in several MCM regions that is also found in the cryptic plasmid pURB500, which was first isolated from $M$. maripaludis C5 (Tumbula et al., 1997). In most cases, the integrase homologue is annotated as a phage integrase (Groth \& Calos, 2004), and is found at varying distances from MCM ORFs. In some cases, such as in the region of MmarC5_1119 (Fig. 3, group 4), the integrase is annotated as a pseudogene; however, a BLASTX search with the nucleotide sequence from this ORF showed that it is a phage integrase homologue $\left(\mathrm{E}=6 \mathrm{e}^{-171}\right)$. Genes found in the $\mathrm{McmA}$ and $\mathrm{McmD}$ groups are not associated with an integrase (Supplementary Fig. S2). A closer examination of the genome context of MCMs falling outside the $\mathrm{McmA}$ and $\mathrm{McmD}$ groups revealed that MCMs that cluster into separate groups in the phylogenetic analysis (groups 1-4, 



Fig. 3. Genome context analysis of Methanococcales MCMs. Analyses suggest that MCMs that fall outside the $m c m A$ and $m c m D$ groups have been generated by duplications involving a phage or mobile element. Groups 1-4 are numbered to correspond with the groups identified in Fig. 2. A phage integrase homologue (yellow) is located close to each MCM (red). MCMs that cluster into separate groups by phylogenetic analysis contain different sets of homologous flanking ORFs, suggesting that several different integration events involving MCMs have occurred. Homologous ORFs within each group are colour coded. ORFs coloured grey show no homology to others in the group. Group $1 \mathrm{MCMs}$ have only two common flanking genes. Group 2 MCMs form two distinct pairs and each is associated with a tRNA gene (vertical black triangles), which is a common site for integration events (Campbell, 1992). Restriction-modification system genes (boxed) are present close to MCMs in group 2 and genes encoding phage structural proteins are present in group 4 (black), providing further evidence for the role of phages in these MCM duplications. Sizes of DNA regions are indicated above each group in base pairs. ORF numbers for MCM and integrase genes are also indicated. Species names are abbreviated as described in the legend to Fig. 2.

Fig. 2) contain different sets of homologous flanking ORFs (Fig. 3), suggesting that several separate integration events involving MCMs have occurred. The presence of homologous flanking genes between groups 3 and 4, and their close phylogenetic relationship indicate that they could have been produced by similar integration events, or by a single integration event followed by a duplication.

\section{Expression and co-purification of $M$. maripaludis S2 MCMs}

The six eukaryotic MCMs form a heterohexameric complex, which is believed to contain one of each of the MCM2-7 proteins (Labib et al., 2000, 2001; Prokhorova \& Blow, 2000). In order to identify whether the multiple MCMs from M. maripaludis S2 could also form a 
heteromeric complex, the four MCM proteins were coexpressed then purified from E. coli. Constructs were generated that allowed the co-expression of all four MCMs, each with a different tag at the $\mathrm{N}$ terminus (His-McmA, TrxHisS-McmB, T7-McmC, GST-McmD). Protein was initially purified by affinity chromatography using glutathione-Sepharose beads, to which GST-tagged $\mathrm{McmD}$ should bind specifically. Western blot analysis of the resulting elution fractions using antibodies specific to each of the tagged MCMs showed that all four MCMs are present in elution fractions 1 and 2 (Fig. 4a). The differences between the levels of signal for each protein seen in the Western blots (Fig. 4) are most likely due to differing antibody specificities, although expression levels and abundance of individual proteins in the isolated complexes may also account for the apparent variation. In order to validate the co-purification of the four $M$. maripaludis S2 MCMs in the single-step GST purification, a multi-step purification was carried out. Protein was purified from cell extract co-expressing all four MCMs first by affinity chromatography using nickel-Sepharose beads, then by size-exclusion chromatography and finally by anion exchange. Western blot analysis of elution fractions from the third purification step showed that all four MCMs were present in the peak anion-exchange fraction 3 (Fig. $4 \mathrm{~b})$. The co-purification of $\mathrm{McmA},-\mathrm{B},-\mathrm{C}$ and $-\mathrm{D}$ through multiple purification steps indicates that the four MCMs form one or more heteromeric complexes in vitro. However, the stoichiometry of the heteromeric complex(es) formed cannot be calculated from these experi- ments, and it is possible that several heteromeric complexes composed of different MCM subunits could be formed.

\section{DISCUSSION}

The Methanococcales are unique among the archaea in possessing multiple apparently functional MCM genes. While a few other archaeal genomes encode more than one MCM sequence, in all cases only one MCM gene possesses all the motifs known to be required for activity (Galagan et al., 2002; McGeoch \& Bell, 2008; Slesarev et al., 2002). The number of MCMs in the Methanococcales ranges from two in M. voltae A3 to eight in M. maripaludis C6. The ML and NJ phylogenies that we have produced are consistent with there being at least two MCM homologues that play important functional roles in the Methanococcales. These two homologues are present in all eight species analysed and appear to have been created by an ancient duplication that pre-dates the divergence of the different Methanococcales species. MCMs that do not fall directly within the $\mathrm{McmA}$ or $\mathrm{McmD}$ groups are all associated with an integrase homologue, suggesting that duplication of these genes is due to the action of a mobile element.

\section{Mechanisms of MCM duplication}

A number of mechanisms for the duplication of integraseassociated MCMs are possible and include (i) the activity of insertion sequence (IS) elements, (ii) transposon activity and (iii) phage-mediated duplication. Although IS ele- (a)

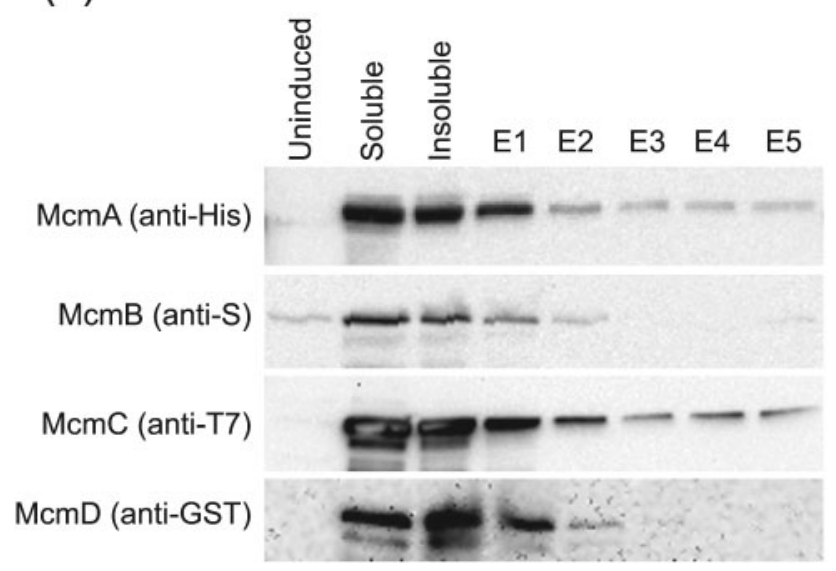

(b)



Fig. 4. All four M. maripaludis MCM proteins co-purify when co-expressed in E. coli. (a) Cells induced to co-express all four $M$. maripaludis S2 MCMs were harvested and lysed to produce a cell-free extract (soluble and insoluble fractions are shown). The soluble fraction was passed over a GST column in order to affinity-purify GST-McmD. The column was extensively washed before $250 \mu$ fractions were eluted using reduced glutathione (E1-E5). Western blots using antibodies specific to each of the differently tagged MCMs (as indicated) showed that all four recombinant MCMs co-purify in this single-step affinity purification. Variability in detection is likely due to varying sensitivity of different antibodies. (b) A purification similar to that above was carried out using a multi-step protocol using nickel-affinity $\left(\mathrm{Ni}^{2+}\right)$ chromatography, size-exclusion chromatography and anion-exchange columns. Samples from $0.5 \mathrm{ml}$ elution fractions from the final column are shown (1-5). Co-elution of all four proteins was detected in fraction 3. 
ments have been identified in Methanocaldococcus jannaschii (Bult et al., 1996), we have been unable to identify any obvious IS elements in the regions where the duplications have occurred. Likewise, searches for repeated sequences characteristic of transposon-type activity have not yielded any noteworthy results. However, the presence of a phage integrase and a smaller associated ORF in the same region as every MCM outside of the McmA and $\mathrm{McmD}$ groups is consistent with a phage-mediated duplication of MCMs in the Methanococcales. tRNA sequences, a common site of phage integration in prokaryotes (Reiter et al., 1989), are present in the same region as a number of integrase-associated MCMs (Fig. 3, group 2), supporting the hypothesis that this duplication was phage-mediated. The presence of putative phage structural proteins (Fig. 3, group 4) and restrictionmodification system genes (Fig. 3, group 2) close to some integrase-associated MCMs is further evidence for the involvement of phages in these MCM duplications. To date, there have been no phages described for the Methanococcales, so there are no obvious candidate phages for involvement in these MCM duplications. However, an association between phage and MCM proteins has been observed in the Bacillus cereus genome, where an MCM homologue is located in an integrated bacteriophage (McGeoch \& Bell, 2005).

\section{Functions of multiple MCMs}

A number of other methanogenic archaea also possess more than one MCM sequence, but in these cases only a single MCM has all of the motifs required for MCM function, suggesting that a single MCM is sufficient for DNA replication and that MCM function in these other duplications has been lost (Bae et al., 2009; McGeoch \& Bell, 2008). Why the Methanococcales are the only order of archaea in which MCM sequences have been duplicated and conserved is not clear. It is possible that only a single MCM is functional, as in other archaea, and that the additional homologues are inactive and not required. However, a shotgun proteomics study carried out in $M$. maripaludis $\mathrm{S} 2$ has detected peptides for $\mathrm{McmA},-\mathrm{B}$ and $-\mathrm{D}$, indicating that multiple MCMs are expressed (Xia et al., 2006). No peptides were detected for McmC, but this does not exclude the possibility of $\mathrm{McmC}$ expression, as peptides may have been present but not detected, or this protein may be expressed only under specific circumstances. Our bioinformatic analysis together with the proteomics data from Xia and colleagues strongly suggest that multiple MCMs are functional in M. maripaludis.

The fact that the MCMs in the Methanococcales have retained the motifs for function suggests either that the duplications have occurred very recently and there has not been sufficient time to accumulate mutations, or that the presence of multiple functional MCMs is in some way advantageous to the cells. Phylogenetic analyses do not support the notion that MCM duplications occurred recently, implying that multiple potentially functional MCM homologues have been retained because they benefit the cell. A codon-based examination of the rates of synonymous versus non-synonymous changes in MCM sequences within a species suggests that a positive selection pressure exists which may be responsible for retaining function in these proteins. In the case of integraseassociated MCMs, it is possible that the advantage in retaining these functions may not be directly cellular, but related to the mobile-element activity associated with these regions. That is, mobile elements are replicated independently of the host genome and require a functional MCM protein to replicate and/or to integrate into the genome. It is also possible that a plasmid-encoded MCM has been integrated into the genome and has been conserved because it is required for plasmid maintenance. Alternatively, multiple MCMs may have been conserved because they provide functions that are beneficial to chromosomal DNA replication. This seems most likely in the case of $\mathrm{McmA}$ and $\mathrm{McmD}$ homologues, which are not integraseassociated and are conserved in all Methanococcales species analysed. While there could be some redundancy among the different MCM homologues, it is also possible that some have diverged to perform specialized functions. In eukaryotes, a degree of specialization has occurred with MCM duplication. The eukaryotic proteins appear to have subtly different roles in the regulation of helicase loading and unwinding (Sheu \& Stillman, 2006; Tsuji et al., 2006), and potentially other functions such as triggering DNA damage checkpoints (Cortez et al., 2004; Tsao et al., 2004). The co-purification of all four recombinant MCMs from M. maripaludis S2 (Fig. 4) indicates that these proteins could form heteromeric complexes with different subunits potentially performing specialized roles.

Both the bioinformatic and in vitro co-purification data that we have produced indicate that the Methanococcales possess a unique complement of MCM proteins that warrants further genetic and biochemical characterization. The multiple potentially functional MCMs in the Methanococcales could provide a model system intermediate between the heteromeric eukaryotic MCM system and the current archaeal systems, where only one MCM is present, for investigating the molecular mechanisms that underlie the enzymic and regulatory roles of different subunits in systems possessing multiple MCMs.

\section{ACKNOWLEDGEMENTS}

Thanks to Jasper Green for help with phylogenetic methods. This work was supported by a UK Biotechnology and Biological Sciences Research Council PhD studentship (to A.D.W.) and a Cancer Research UK grant (C23949/A7771) to J. P. J.C.

\section{REFERENCES}

Altschul, S. F., Madden, T. L., Schaffer, A. A., Zhang, J., Zhang, Z., Miller, W. \& Lipman, D. J. (1997). Gapped BLAST and PSI-BLAST: a new 
generation of protein database search programs. Nucleic Acids Res 25, 3389-3402.

Bae, B., Chen, Y. H., Costa, A., Onesti, S., Brunzelle, J. S., Lin, Y., Cann, I. K. \& Nair, S. K. (2009). Insights into the architecture of the replicative helicase from the structure of an archaeal MCM homolog. Structure 17, 211-222.

Bailey, T. L. \& Elkan, C. (1994). Fitting a mixture model by expectation maximization to discover motifs in biopolymers. Proc Int Conf Intell Syst Mol Biol 2, 28-36.

Barry, E. R., McGeoch, A. T., Kelman, Z. \& Bell, S. D. (2007). Archaeal MCM has separable processivity, substrate choice and helicase domains. Nucleic Acids Res 35, 988-998.

Bult, C. J., White, O., Olsen, G. J., Zhou, L., Fleischmann, R. D., Sutton, G. G., Blake, J. A., FitzGerald, L. M., Clayton, R. A. \& other authors (1996). Complete genome sequence of the methanogenic archaeon, Methanococcus jannaschii. Science 273, 1058-1073.

Burland, T. G. (2000). DNASTAR's Lasergene sequence analysis software. Methods Mol Biol 132, 71-91.

Campbell, A. (1992). Chromosomal insertion sites for phages and plasmids. J Bacteriol 174, 7495-7499.

Carpentieri, F., De Felice, M., De Falco, M., Rossi, M. \& Pisani, F. M. (2002). Physical and functional interaction between the minichromosome maintenance-like DNA helicase and the single-stranded DNA binding protein from the crenarchaeon Sulfolobus solfataricus. J Biol Chem 277, 12118-12127.

Castresana, J. (2000). Selection of conserved blocks from multiple alignments for their use in phylogenetic analysis. Mol Biol Evol 17, 540-552.

Chong, J. P., Hayashi, M. K., Simon, M. N., Xu, R. M. \& Stillman, B. (2000). A double-hexamer archaeal minichromosome maintenance protein is an ATP-dependent DNA helicase. Proc Natl Acad Sci U S A 97, 1530-1535.

Cortez, D., Glick, G. \& Elledge, S. J. (2004). Minichromosome maintenance proteins are direct targets of the ATM and ATR checkpoint kinases. Proc Natl Acad Sci U S A 101, 10078-10083.

Edgell, D. R. \& Doolittle, W. F. (1997). Archaea and the origin(s) of DNA replication proteins. Cell 89, 995-998.

Galagan, J. E., Nusbaum, C., Roy, A. \& other authors (2002). The genome of $M$. acetivorans reveals extensive metabolic and physiological diversity. Genome Res 12, 532-542.

Grainge, I., Scaife, S. \& Wigley, D. B. (2003). Biochemical analysis of components of the pre-replication complex of Archaeoglobus fulgidus. Nucleic Acids Res 31, 4888-4898.

Groth, A. C. \& Calos, M. P. (2004). Phage integrases: biology and applications. J Mol Biol 335, 667-678.

Guindon, S. \& Gascuel, O. (2003). A simple, fast, and accurate algorithm to estimate large phylogenies by maximum likelihood. Syst Biol 52, 696-704.

Hendrickson, E. L., Kaul, R., Zhou, Y. \& other authors (2004). Complete genome sequence of the genetically tractable hydrogenotrophic methanogen Methanococcus maripaludis. J Bacteriol 186, 6956-6969.

Jenkinson, E. R. \& Chong, J. P. (2006). Minichromosome maintenance helicase activity is controlled by $\mathrm{N}$ - and C-terminal motifs and requires the ATPase domain helix-2 insert. Proc Natl Acad Sci U S A 103, 7613-7618.

Jones, D. T., Taylor, W. R. \& Thornton, J. M. (1992). The rapid generation of mutation data matrices from protein sequences. Comput Appl Biosci 8, 275-282.

Kasiviswanathan, R., Shin, J. H., Melamud, E. \& Kelman, Z. (2004). Biochemical characterization of the Methanothermobacter thermauto- trophicus minichromosome maintenance (MCM) helicase N-terminal domains. J Biol Chem 279, 28358-28366.

Kelman, Z., Lee, J. K. \& Hurwitz, J. (1999). The single minichromosome maintenance protein of Methanobacterium thermoautotrophicum $\Delta \mathrm{H}$ contains DNA helicase activity. Proc Natl Acad Sci U S A 96, 14783-14788.

Labib, K., Tercero, J. A. \& Diffley, J. F. (2000). Uninterrupted MCM27 function required for DNA replication fork progression. Science 288, 1643-1647.

Labib, K., Kearsey, S. E. \& Diffley, J. F. (2001). MCM2-7 proteins are essential components of prereplicative complexes that accumulate cooperatively in the nucleus during G1-phase and are required to establish, but not maintain, the S-phase checkpoint. Mol Biol Cell 12, 3658-3667.

Lin, D. I., Aggarwal, P. \& Diehl, J. A. (2008). Phosphorylation of MCM3 on Ser-112 regulates its incorporation into the MCM2-7 complex. Proc Natl Acad Sci U S A 105, 8079-8084.

McGeoch, A. T. \& Bell, S. D. (2005). Eukaryotic/archaeal primase and MCM proteins encoded in a bacteriophage genome. Cell 120, 167168.

McGeoch, A. T. \& Bell, S. D. (2008). Extra-chromosomal elements and the evolution of cellular DNA replication machineries. Nat Rev Mol Cell Biol 9, 569-574.

Overbeek, R., Begley, T., Butler, R. M. \& other authors (2005). The subsystems approach to genome annotation and its use in the project to annotate 1000 genomes. Nucleic Acids Res 33, 5691-5702.

Poplawski, A., Grabowski, B., Long, S. E. \& Kelman, Z. (2001). The zinc finger domain of the archaeal minichromosome maintenance protein is required for helicase activity. J Biol Chem 276, 4937149377.

Prokhorova, T. A. \& Blow, J. J. (2000). Sequential MCM/P1 subcomplex assembly is required to form a heterohexamer with replication licensing activity. J Biol Chem 275, 2491-2498.

Pruitt, K. D., Tatusova, T. \& Maglott, D. R. (2007). NCBI reference sequences (RefSeq): a curated non-redundant sequence database of genomes, transcripts and proteins. Nucleic Acids Res 35, D61-D65.

Reiter, W. D., Palm, P. \& Yeats, S. (1989). Transfer RNA genes frequently serve as integration sites for prokaryotic genetic elements. Nucleic Acids Res 17, 1907-1914.

Shechter, D. F., Ying, C. Y. \& Gautier, J. (2000). The intrinsic DNA helicase activity of Methanobacterium thermoautotrophicum $\Delta \mathrm{H}$ minichromosome maintenance protein. J Biol Chem 275, 1504915059 .

Sheu, Y. J. \& Stillman, B. (2006). Cdc7-Dbf4 phosphorylates MCM proteins via a docking site-mediated mechanism to promote $S$ phase progression. Mol Cell 24, 101-113.

Shi, Y., Dodson, G. E., Mukhopadhyay, P. S., Shanware, N. P., Trinh, A. T. \& Tibbetts, R. S. (2007). Identification of carboxyl-terminal MCM3 phosphorylation sites using polyreactive phosphospecific antibodies. J Biol Chem 282, 9236-9243.

Slesarev, A. I., Mezhevaya, K. V., Makarova, K. S. \& other authors (2002). The complete genome of hyperthermophile Methanopyrus kandleri AV19 and monophyly of archaeal methanogens. Proc Natl Acad Sci U S A 99, 4644-4649.

Takei, Y., Assenberg, M., Tsujimoto, G. \& Laskey, R. (2002). The MCM3 acetylase MCM3AP inhibits initiation, but not elongation, of DNA replication via interaction with MCM3. J Biol Chem 277, 4312143125.

Thauer, R. K. (1998). Biochemistry of methanogenesis: a tribute to Marjory Stephenson. 1998 Marjory Stephenson Prize Lecture. Microbiology 144, 2377-2406. 
Thompson, J. D., Gibson, T. J., Plewniak, F., Jeanmougin, F. \& Higgins, D. G. (1997). The CLUSTAL_X Windows interface: flexible strategies for multiple sequence alignment aided by quality analysis tools. Nucleic Acids Res 25, 4876-4882.

Tsao, C. C., Geisen, C. \& Abraham, R. T. (2004). Interaction between human MCM7 and Rad17 proteins is required for replication checkpoint signaling. EMBO J 23, 4660-4669.

Tsuji, T., Ficarro, S. B. \& Jiang, W. (2006). Essential role of phosphorylation of MCM2 by Cdc7/Dbf4 in the initiation of DNA replication in mammalian cells. Mol Biol Cell 17, 4459-4472.

Tumbula, D. L., Bowen, T. L. \& Whitman, W. B. (1997). Characterization of pURB500 from the archaeon Methanococcus maripaludis and construction of a shuttle vector. J Bacteriol 179, 2976-2986.
Tye, B. K. (2000). Insights into DNA replication from the third domain of life. Proc Natl Acad Sci U S A 97, 2399-2401.

Walters, A. D. \& Chong, J. P. (2009). Methanococcus maripaludis: an archaeon with multiple functional MCM proteins? Biochem Soc Trans 37, 1-6.

Woese, C. R. \& Fox, G. E. (1977). Phylogenetic structure of the prokaryotic domain: the primary kingdoms. Proc Natl Acad Sci U S A 74, 5088-5090.

Xia, Q., Hendrickson, E. L., Zhang, Y. \& other authors (2006). Quantitative proteomics of the archaeon Methanococcus maripaludis validated by microarray analysis and real time PCR. Mol Cell Proteomics 5, 868-881.

Edited by: D. W. Ussery 